\title{
Association of Genetic Polymorphism of Insulin Receptor Substrate 1 (IRS-1) with Polycystic Ovary Syndrome Pathogenicity in Iraqi Women
}

\author{
Zahraa Fouad Fadhil $^{1}$, Ban Hoshi khalaf ${ }^{2}$, Hassan Mahmoud Mousa Abo Almaali ${ }^{3}$ \\ ${ }^{1}$ B.Sc. Pharmacy, Candidate of Master of Science in Pharmacology and Toxicology, Department of Pharmacology \\ and Toxicology, College of Pharmacy, Kerbala University, Iraq, ${ }^{2}$ PhD, Professor in Pharmacology and Toxicology, \\ Department of Pharmacology and Toxicology, College of Pharmacy, Kerbala University, Iraq, ${ }^{3}$ PhD, Assistant \\ Professor of Genetic Engineering and Biotechnology, Branch of Clinical Laboratory Sciences College of \\ Pharmacy, Kerbala University, Iraq
}

\begin{abstract}
Background: Insulin receptor substrate 1 (IRS-1) is an intracellular signaling adapter protein that integrates and coordinates multiple biologically key extracellular signals within the cell, is also a key central receptor in insulin signaling, and plays a focal role in maintaining essential cellular capabilities, e.g., survival, development and digestion system. IRS1 is essentially found in the cytoplasm But localization in nucleus may occur in some cell types and under certain stimuli.
\end{abstract}

Materials and Method: A total of 104 healthy control and 215 Iraqi women have Polycystic ovary syndrome (PCOS) aged 20-40 years who was admitted to conducted a prospective clinical study at kerbala gynecology teaching hospital, it was measured the genotype distribution of the rs2943641 T to C substitution of IRS1 and the effects of genotypes on Polycystic Ovary syndrome Pathogenicity in Iraqiwomen,

Results: Analyses were conducted to assess the association between the SNP rs2943641 [TT (Wild type), TC (heterozygous type), and CC (mutated type)], with the pathogenesis of PCOS according to logistic regression results. This survey demonstrated that there was no significant association between different alleles for this SNP with the pathogenesis of PCOS

Conclusion: Genetic polymorphism with IRS-1may be associated with metabolic disturbance but not Polycystic Ovary syndrome in Iraqiwomen.

Keywords: Polycystic Ovary Syndrome, Pathogenicity, Genetic Polymorphism, Insulin Receptor Substrate 1.

\section{Introduction}

Polycystic ovary syndrome (PCOS) is highly prevalent hormonal disorder among reproductive-aged women. Its clinical manifestations are heterogeneous ${ }^{(1)}$.

\section{Corresponding Author:}

\section{Zahraa Fouad Fadhil}

B.Sc. Pharmacy, Candidate of Master of Science in Pharmacology and Toxicology, Department of Pharmacology and Toxicology, College of Pharmacy, Kerbala University, Iraq e-mail: zahraazozo1221@gmail.com at a recent time, they are different studies have proven that genetic factors are associated with the occurrence of PCOS, although many candidate genetic mutations and single nucleotide polymorphisms (SNPs) have been studied for poly cystic ovary ${ }^{(2)}$, the molecular technique subordinate with PCOS, and the mode of inheritance for PCOS was unclear, in addition to the scarcity of sources on the subject of insulin receptor substrate 1 (IRS1) with PCOS, It was also considered as novel candidate gene for $\mathrm{PCOS}^{(1)}$. The IRS1 is the most important intermediate in insulin signaling and plays a crucial role in maintaining the essential function of the cell, so any polymorphism in IRS gene acts as a competitive inhibitor of the insulin receptors. polymorphism in IRS1 gene lead to 
susceptibility to insulin resistance and PCOS, Molecular scanning of the IRS1 gene has showed substitutions of several amino acid ${ }^{(3)}$. Insulin Receptor Substrate 1 (IRS1) polymorphism significantly decrease insulin-dependent receptor tyrosine autophosphorylation and increase Insulin-independent receptor serine phosphorylation markedly ${ }^{(3)}$. These serine phosphorylation inhibit normal receptor signaling and make the primary defects in insulin-stimulated glucose transporters (GLUT4) production $^{(4)}$, Decreased glucose uptake may result from suppressed insulin signaling or impaired glucose transporter (GLUT) 4 trafficking. In adipocytes of women with PCOS that decrease insulin responsiveness ${ }^{(5)}$.

\section{Materials and Method}

Sample Collection: A total of 104 healthy control and 215 Iraqi women with PCOS, aged 20-40 years old have been recorded the PCOS subjects had at least two of the following signs : 1) chronic oligoanovulation 2) hirsutism or increased serum total testosterone levels; and 3) polycystic ovarian morphology at ultrasound, according to the Rotterdam consensus. Conference criteria (6). The protocol was approved by the local Ethics Committee, and all women gave written informed consent.

Polymerase chain reaction: The human genomic DNA extracted from whole blood by using genomic DNA extraction kit (G-DEX llb Introne, korea), according to the manufacture company, the purity and concentration of DNA obtaining was determinate by nanodrop apparatus (biobase, china), Polymorphism of IRS1 gene was detected by amplification refractory mutation system (ARMS) polymerase chain reaction
(PCR),nucleotides primers were designed through Primer-BLAST allows users to design new targetspecific primers according to the websites (https://www. ncbi.nlm.nih.gov/tools/primer-blast/index.cgi) it is also prepared by (Bioneercom.Korea) company as the following (Table 1)

Optimization of PCR reaction was recorded as initial denaturation for $3 \mathrm{~min}$ at $94{ }^{\circ} \mathrm{C}$, followed by 35 cycles consist of second denaturation for at $94{ }^{\circ} \mathrm{C}$ 30 seconds, 45 second at $56{ }^{\circ} \mathrm{C}$, first extension 55 seconds at $72{ }^{\circ} \mathrm{C}$, then last extension at $72{ }^{\circ} \mathrm{C}$ for five minutes. The amplification of insulin receptor substrate 1. was run electrophoresis in the $1.5 \%$ concentration of agarose gel at $70 \mathrm{~V}$ for 60 min after stained with $2 \mu \mathrm{L}$ ethidium bromide, the product size was visualized under Ultraviolet.

Statistical Analysis: Statistical analysis were used by software SPSS program version 20, Test for HardyWeinberg equilibrium in controls and allelic or genotypic association in cases versus control were evaluated by Chi - square $\left(\mathrm{x}^{2}\right)$ test. This analysis was performed for all genotypes in this study using Hardy-Weinberg equilibrium online calculator.To assess the predictability of PCOS, logistic analysis of SNP was applied, this yielded odds ratio (OR) also the $95 \%$ confidence interval of the OR was calculated which is good estimator for the significance of the OR; when the value of "one" included within interval, this is an indicator that the OR is not significant. All statistical procedures and tests were applied under a level of significance (P- value) of less than 0.05 to be considered as significant difference or correlation.

Table 1. ARMS PCR primer nucleotides used in this study with product size

\begin{tabular}{|c|c|c|c|}
\hline Primers & & Sequence & Product size (bp) \\
\hline \multirow{4}{*}{$\begin{array}{l}\text { Primers sequences } \\
\text { of IRS1 rs } 2943641 \\
\text { Alleles }>\text { C }\end{array}$} & $\mathrm{O}-\mathrm{F}$ & TGGTTCTGTAACTGGGTG & 537 \\
\hline & O-R & AGTTGAAGTAGCCATCTTTC & 537 \\
\hline & Allele T & ATCAGGGCTAATAGTTAGAAGA & 387 \\
\hline & Allele C & GTTGGAAATGAGAGGAACC & 190 \\
\hline
\end{tabular}

\section{Results and Discussion}

PCOS is a polygenic endocrine and metabolic disorder. The prevalence of PCOS has grown rapidly.
Several genetic polymorphisms have already been enrolled in the pathogenesis of $\operatorname{PCOS}^{(7)}$. The IRS-1 gene has been considered to be a candidate gene for the etiology of metabolic diseases such as type $2 \mathrm{DM}$, 
PCOS, and obesity. The presence of polymorphisms of the IRS-1 gene has been documented to be associated with the development of IR. The IRS-1 gene located on chromosome $2 q 36$ is the substrate for the insulin tyrosine kinase receptor, responsible for insulin signaling. The protein is expressed in multiple cells and tissues sensitive to insulin. Binding of insulin to its receptor activates phosphorylation of cytosolic substrates of IRS-1 ${ }^{(8)}$. IRS-1 activation is a first step in the insulin signaling pathway, and functional studies of polymorphism in the IRS-1 gene showed weak insulin signals through the PI3-kinase pathway ${ }^{(9)}$.

Analyses were conducted to assess the association between the SNP rs2943641 with the pathogenesis of PCOS according to logistic regression and (Figure 1) . This survey demonstrated that there was no significant association between different alleles for this SNP with the pathogenesis of PCOS, (Tables 2 and 3).

The exact cause of PCOS is unknown, but several studies suggest a strong genetic component that is affected by gestational environment and lifestyle factors, or both ${ }^{(10)}$. Thus, numerous genetic variations have been related to the presence of PCOS in different populations $^{(11)}$. In the present study, we investigated the possible association between the single nucleotide polymorphisms (SNPs) (rs2943641) of the IRS1 gene and susceptibility to PCOS in Iraqi women, (TT wild type, TC heterozygotes, and $\mathrm{CC}$ mutated form for the two SNPs). The frequencies of SNP rs2943641 variant observed in our study were not significantly different between PCOS and healthy control women (11.5\% vs. $12.1 \%, \mathrm{P}=0.368)$ as shown in table (2). Our data reviled that the IRS-1 polymorphism (rs2943641) is not associated with increased susceptibility to PCOS in Iraqi populations. However, we cannot exclude the possibility that other genetic polymorphisms of the IRS1 family are associated with PCOS and might be clinically useful as markers to assess the disease risk, as polymorphism of Gly972Arg that could play a contributory role in the pathophysiology and risk of PCOS ${ }^{(12)}$. But C allele of rs2943641 is associated with increased hyperinsulinemia and impaired insulin sensitivity ${ }^{(13)}$.

Table 2. Distribution of SNP rs2943641 in the healthy control group and polycystic ovary syndromegroup.

\begin{tabular}{|l|c|c|c|}
\hline Variables & Control & PCOS & p-value \\
\hline Number & 104 & 215 & - \\
\hline SNP1 (rs2943641) & & & \multirow{2}{*}{0.368} \\
\cline { 1 - 3 } TT (Wild type) & $24(23.1 \%)$ & $65(30.2 \%)$ & \multirow{2}{*}{$124(57.7 \%)$} \\
\hline TC (Heterozygotes) & $68(65.4 \%)$ & $26(12.1 \%)$ & \\
\hline CC (Mutant) & $12(11.5 \%)$ & & \\
\hline
\end{tabular}

Table 3. Logistic analysis of SNP rs2943641 to predict polycystic ovary syndrome pathogenesis.

\begin{tabular}{|l|c|c|}
\hline SNP1 (rs2943641) & OR $\mathbf{( 9 5 \% C I )}$ & p-value \\
\hline TT (Wild type) & 1.0 (reference value) & - \\
\hline TC & $0.67(0.39-1.17)$ & 0.161 \\
\hline CC & $0.80(0.35-1.83)$ & 0.598 \\
\hline
\end{tabular}

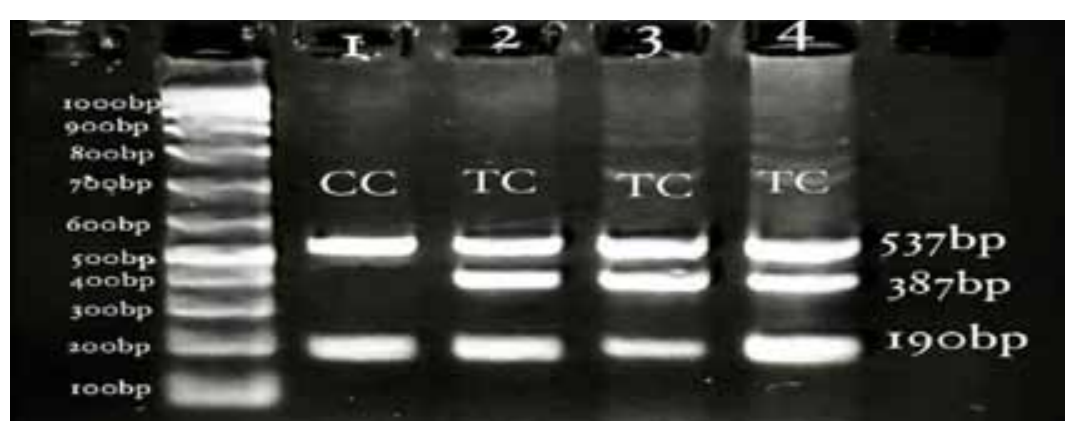

Figure 1. ARMS-PCR amplification of IRS1 gene $T>C$ showing the outer primer $537 \mathrm{bp}$ in size, $T$ allele is $387 \mathrm{bp}$ in size while $\mathrm{C}$ allele is $190 \mathrm{bp}$ in size. 


\section{Conclusion}

Polycystic ovary syndrome is associated with hyperinsulinemia and insulin resistance that affected by insulin receptor substrate 1 (IRS1) protein, this protein is an important intermediate in insulin signaling and plays a key role in maintaining the basic function of the cell, so any polymorphism in IRS1 genes acts as a competitive inhibitor of the insulin receptor. IRS1 rs2943641 polymorphism may associated with susceptibility to insulin resistance but not poly cystic ovary syndrome in Iraqi women.

Ethical Clearance: Informed consent was obtained from all participants, Data were collected in accordance with declaration of Helsinki of the World Medical Association, 2013, all other ethical issues were approved by the authors from the University of Kerbala

Conflict of Interest: Authors Declared none.

Funding: None

Self-funded by corresponding author.

\section{Reference}

1. Ovalle F, Azziz R. Insulin resistance, polycystic ovary syndrome, and type 2 diabetes mellitus. Fertility and sterility. 2002;77(6):1095-105.

2. Dumesic DA, Oberfield SE, Stener-Victorin E, Marshall JC, Laven JS, Legro RS. Scientific statement on the diagnostic criteria, epidemiology, pathophysiology, and molecular genetics of polycystic ovary syndrome. Endocrine reviews. 2015;36(5):487-525.

3. Boucher J, Kleinridders A, Kahn CR. Insulin receptor signaling in normal and insulin-resistant states. Cold Spring Harbor perspectives in biology. 2014;6(1):a009191.

4. Diamanti-KandarakisE, DunaifA. Insulin resistance and the polycystic ovary syndrome revisited: an update on mechanisms and implications. Endocrine reviews. 2012;33(6):981-1030.
5. Karnieli E, Armoni M. Transcriptional regulation of the insulin-responsive glucose transporter GLUT4 gene: from physiology to pathology. American Journal of Physiology-Endocrinology and Metabolism. 2008;295(1):E38-E45.

6. Hiam D. The Molecular Basis of Polycystic Ovary Syndrome: Genetics, Epigenetics and Insulin Resistance: Victoria University; 2018; 2:1010-15.

7. Conway G, Dewailly D, Diamanti-Kandarakis E, Escobar-Morreale HF, Franks S, Gambineri A, et al. The polycystic ovary syndrome: a position statement from the European Society of Endocrinology. European journal of endocrinology. 2014;171(4):P1-P29.

8. Pandey SK, Yu XX, Watts LM, Michael MD, Sloop KW, Rivard AR, et al. Reduction of low molecular weight protein-tyrosine phosphatase expression improves hyperglycemia and insulin sensitivity in obese mice. Journal of Biological Chemistry. 2007;282(19):14291-9.

9. Avogaro A, de Kreutzenberg SV, Fadini GP. Insulin signaling and life span. Pflügers Archiv-European Journal of Physiology. 2010;459(2):301-14.

10. Norman RJ, Dewailly D, Legro RS, Hickey TE. Polycystic ovary syndrome. The Lancet. 2007;370(9588):685-97.

11. Diamanti-Kandarakis E, Piperi C. Genetics of polycystic ovary syndrome: searching for the way out of the labyrinth. Human Reproduction Update. 2005;11(6):631-43.

12. Thangavelu M, Godla UR, Paul SF, Maddaly R. Single-nucleotide polymorphism of INS, INSR, IRS1, IRS2, PPAR-G and CAPN10 genes in the pathogenesis of polycystic ovary syndrome. Journal of genetics. 2017;96(1):87-96.

13. Rung J, Cauchi S, Albrechtsen A, Shen L, Rocheleau G, Cavalcanti-Proença C, et al. Genetic variant near IRS1 is associated with type 2 diabetes, insulin resistance and hyperinsulinemia. Nature genetics. 2009;41(10):1110. 